\title{
High-Energy Photoproduction Amplitudes from a New Kind of Dispersive Sum Rules.
}

\author{
P. Gensini \\ Istituto di Fisica dell'Università - Lecce \\ Istituto Nazionale di Fisica Nucleare - Sottosezione di Bari \\ (Lett. Nuovo Cimento, 2, 381 (1969))
}

In Table I of this paper some errors have crept in : the correct Table is printed below.

TABLE I.

\begin{tabular}{|c|c|c|c|}
\hline$t\left((\mathrm{GeV})^{2}\right)$ & $\gamma_{2}(t)\left(\mu \mathrm{b}^{\frac{1}{2}}\right)$ & $\gamma_{3}(t)\left(\mu \mathrm{b}^{\frac{1}{2}} \cdot \mathrm{GeV}\right)$ & \multicolumn{1}{c|}{$\beta(t)\left(\mu b^{\frac{1}{2}}\right)$} \\
\hline & $a$ & $b$ & $c$ \\
-0.15 & $7.25 \pm 2.18$ & $-27.46 \pm 4.28$ & $0.77 \pm 0.12$ \\
-0.10 & $3.97 \pm 0.38$ & $-9.75 \pm 2.44$ & $0.40 \pm 0.10$ \\
-0.05 & $1.56 \pm 0.13$ & $-1.05 \pm 0.92$ & $0.08 \pm 0.07$ \\
0.00 & $-0.79 \pm 0.22$ & $1.20 \pm 0.25$ & $-0.64 \pm 0.12$ \\
0.05 & $-2.78 \pm 0.21$ & $-6.28 \pm 2.34$ & $-0.67 \pm 0.25$ \\
0.10 & $-4.02 \pm 2.20$ & $-27.9 \pm 16.1$ & $-1.35 \pm 0.78$ \\
0.15 & $-6.59 \pm 2.10$ & $-15.1 \pm 15.1$ & $-0.94 \pm 0.94$ \\
\hline
\end{tabular}

